\title{
Short-Term Clinical and Oncological Outcome of Prolonging Operation Interval After Neoadjuvant Chemoradiotherapy for Locally Advanced Middle and Low Rectal Cancer
}

This article was published in the following Dove Press journal:

Cancer Management and Research

\section{Jianguo Yang \\ Qingwei Chen \\ Jindou $\mathrm{Li}$ \\ Zhiyang Song \\ Yong Cheng}

Department of Gastrointestinal Surgery, First Affiliated Hospital of Chongqing Medical University, Chongqing, People's Republic of China
Correspondence: Yong Cheng Department of Gastrointestinal Surgery, First Affiliated Hospital of Chongqing Medical University, Chongqing, People's Republic of China

Email Chengyongcq@I63.Com
Purpose: The purpose of this study is to evaluate the short-term clinical and oncological outcome of prolonging operation interval to 11 weeks after the end of radiotherapy for locally advanced middle and low rectal cancer.

Methods: A total of 123 patients with stage II/III (cT3/T4 or N+) low and middle rectal cancer who had undergone operation after neoadjuvant chemoradiotherapy were selected. According to the interval time between the last radiotherapy and operation, they were assigned to a short-interval group ( $\mathrm{SG},<11$ weeks, $\mathrm{n}=66$ ) and long-interval group (LG, $\geq 11$ weeks, $\mathrm{n}=57$ ). The relations among interval time and short-term clinical outcome and oncological outcome were analyzed.

Results: The analysis found that basic information, clinical characteristics, and preoperative treatment between the two groups had no significant difference. There were no differences in operation time, estimated intraoperative blood loss and postoperative complications. The rate of sphincter preservation in the low and middle rectum was $66.7 \%$ in the short-interval group and $59.7 \%$ in the long-interval group $(P=0.42)$. The incidence of anastomotic leak in the long-interval group was higher than that in the short-interval group $(P=0.08)$. There was no significant difference in the recovery time of intestinal function and median duration of hospitalization between the two groups. The pathological complete remission rate was $17.07 \%$. Multivariate analysis showed interval time had no influence on pathological complete remission. There was no significant difference in 3-year overall survival and 3-year disease-free survival between the two groups. The risk of recurrence and metastasis in patients with positive lymph nodes was higher than those with negative lymph nodes $(P<0.05), \mathrm{HR}=4.812$ (95\% CI 2.4-9.648).

Conclusion: Prolonging the interval time of operation to 11 weeks after neoadjuvant chemoradiotherapy for middle and low rectal cancer does not improve the pathologic complete remission, morbidity, and mortality. There was no significant effect on oncologic outcome after prolonging the operation interval. Therefore, it is safe to prolong the interval of operation to 11 weeks.

Keywords: rectal cancer, neoadjuvant chemoradiotherapy, oncological outcome, clinical outcome, pathological complete remission

\section{Introduction}

In recent years, the incidence of colorectal cancer (CRC) has increased year by year. $\mathrm{CRC}$ has become the second most common cause of cancer-related death. ${ }^{1}$ Many patients in China suffer from locally advanced rectal cancer (LARC) when they are 
diagnosed. Total mesorectal excision (TME) operation after neoadjuvant chemoradiotherapy (nCRT) has become the standard treatment regimen for LARC. Neoadjuvant chemoradiotherapy can effectively control local tumors, make tumors shrink, increase sphincter preservation rate, and reduce local recurrence of tumors. Compared with postoperative radiotherapy and chemotherapy, nCRT can improve the prognosis of patients. ${ }^{2-6}$ However, the time interval between the end of radiotherapy and TME operation remains controversial.

In 1999, the Lyon R90-01 randomized trial divided patients with LARC into 2 weeks and 6-8 weeks after nCRT. The results showed that the 6-8 weeks group had better tumor regression, downstage, sphincter preservation rate, and pathological complete remission ( $\mathrm{pCR}$ ) rate than the 2-week group. ${ }^{7}$ Since then, the interval of 6-8 weeks has been considered as the most appropriate time for operation after nCRT. The distant control, overall survival (OS), and disease-free survival (DFS) of patients with rectal cancer after nCRT have been improved, and some of them can achieve pCR. Pathological complete remission is closely correlated with local control, DFS, and OS. ${ }^{8,9,18-21}$ Studies have shown that prolonging the interval between the last radiotherapy and operation can achieve pathological complete remission rate, but does not increase complications. ${ }^{10,15,16}$ Tumor regression is linked with time. The interval of operation recommended in NCCN guidelines for rectal cancer after long-term radiotherapy is also changing, from the original 4-6 weeks to 5-12 weeks currently. ${ }^{11}$ In addition, the Chinese Criteria for Diagnosis and Treatment of Colorectal Cancer also recommends 5-12 weeks as a reasonable interval after neoadjuvant chemoradiotherapy for stage II and III rectal cancer, which is consistent with NCCN guidelines. ${ }^{12}$ It is obvious that prolonging the operation interval properly can lead to tumor regression further, even achieving complete remission. Prolonging the interval time can also help to improve the R0 resection rate, and reduce the positive rate of circumferential resection margin (CRM). Patients who have a strong desire for sphincter preservation with clinic complete remission have the opportunity to be selected in a "watch \& wait" program (W\&W). Nevertheless, studies have shown that prolonging the time interval after nCRT did not improve tumor remission and pathological complete response rate, reduce the incidence of postoperative complications and another adverse short-term clinical outcome instead. ${ }^{13,14}$

So far, there is no definite conclusion about the time interval of operation after neoadjuvant chemoradiotherapy.
With extension of time interval, the tissue fibrosis in the radiotherapy area can become severe, which can affect surgery. Planar fibrosis, severe edema, and local inflammation enhance difficulties in TME operation and complications of operation and reduce the $\mathrm{R} 0$ resection rate. ${ }^{17,26,27}$ In addition, the prolongation of the time interval may increase the risk of local progression and distant metastasis, which can lead to patients missing the optimal operation time.

A prospective study showed that prolonging the operation time to 11 weeks after nCRT did not increase pCR, but caused higher intraoperative and postoperative morbidity. The 11-week group had a worse quality of TME than the 7-week group. ${ }^{13}$ Therefore, we conducted a singlecenter retrospective study to explore the effect of extending the interval time to 11 weeks on short-term clinical outcome and tumor outcome.

\section{Method}

Patients with rectal cancer who experienced neoadjuvant chemoradiotherapy and radical surgical resection from 2010 to 2016 were selected from the First Affiliated Hospital of Chongqing Medical University. Preoperative, postoperative and follow-up data were collected.

\section{Patient Selection}

Altogether 148 patients with locally advanced rectal cancer $\left(\mathrm{cT}_{3} / \mathrm{T}_{4}\right.$ or $\left.\mathrm{N}^{+}\right)$who had undergone neoadjuvant chemoradiotherapy were selected from 2000 rectal cancer patients in the First Affiliated Hospital of Chongqing Medical University. Among them, there were 10 patients with high rectal cancer, 6 patients without follow-up after operation, 1 patient with rectal squamous carcinoma, 3 patients with signet ring cell cancer, 2 patients without operation because they could not preserve anal sphincter, one with distant metastasis during neoadjuvant chemoradiotherapy and 2 patients without inadequate preoperative data. Finally, 25 patients were excluded, and 123 patients were included. All patients included were over 18 years old.

\section{Evaluation Before Neoadjuvant Chemoradiotherapy}

Routine tumor estimation and physical examination before nCRT were carried out. Rectal adenocarcinoma (including mucinous adenocarcinoma) was confirmed by colonoscopy and pathological examination before operation. A computed tomography (CT) scan of chest, abdomen, and pelvis excluded 
distant organ metastases, such as liver and lung metastases. High-resolution magnetic resonance imaging (MRI) of the pelvis was used to evaluate the local rectal tumors, which contained $\mathrm{T}$ and $\mathrm{N}$ stage of rectal tumors according to AJCC (American Joint Committee on Cancer Stage), long diameter, and the distance of anal verge, separation, circumferential resection margin (CRM), and extramural vascular invasion (EMVI).

\section{Process on Neoadjuvant Chemoradiotherapy}

The long-course radiotherapy with a dose of 45-50.4 Gy was divided into 25-28 times, 1.8-2.0 Gy each time, which was completed within five and a half weeks. Capecitabine at $825 \mathrm{mg} / \mathrm{m}^{2}$ was given orally twice a day during radiotherapy. Some patients received standard capecitabine and oxaliplatin neoadjuvant chemotherapy (nCT) in the period of interval time. Each cycle consisted of intravenous oxaliplatin with $130 \mathrm{mg} / \mathrm{m}^{2}$ on day 1 , and oral administration capecitabine with $1000 \mathrm{mg} / \mathrm{m}^{2}$ twice on day 1 to day 14 .

\section{Preoperative Assessment}

All patients went through a digital rectal examination before surgery. The enhanced CTs of chest, abdomen, and pelvis were used to exclude distant organ metastasis. High-resolution magnetic resonance imaging (MRI) was used to re-evaluate local rectal tumors, including $\mathrm{T}$ stage, $\mathrm{N}$ stage, Mandard Tumor Regression Grading (TRG), CRM, and EMVI.

\section{Outcome Assessment}

The baseline characteristics and surgical outcomes of patients who underwent nCRT and radical surgical operations were analyzed. Baseline characteristics included gender, age, carcinoembryonic antigen (CEA), carbohydrate antigen 19-9 (CA19-9), body mass index (BMI), tumor size, tumor location, pathological type of tumor, differentiation degree of tumor, ASA (American Society of Anesthesiologists) grading, T stage, N stage, CRM, EMVI, neoadjuvant chemotherapy, and preoperative TRG grading. Perioperative data were collected including operation time, estimated blood loss, operation method (laparotomy vs laparoscope vs robot) and operation mode. Postoperative clinical data included hospitalization time, anal exsufflation time, anal defecation time, eating liquid diet time, drainage tube extraction time, 30 days readmission, 30 days re-operation, 30 days mortality rate, anastomotic leakage, and other postoperative complications. Postoperative pathological data included $\mathrm{T}$ stage,
$\mathrm{N}$ stage, number of positive lymph nodes, ypTRG grading (AJCC tumor regression grading).

\section{Follow-Up}

Patients accepted follow-up every 3-6 months in the first 2 years after operation. In each follow-up, patients received a series of examinations, including digital rectal examination, blood count, blood chemistries, and digestive tract cancer spectrum (including CEA, CA19-9, and AFP). Enhanced computed tomography (CT) of chest, abdomen, and pelvis was performed every 6 months. Colonoscopy was performed every 1-2 years after operation. Three-year disease-free survival and overall survival were also collected. Disease-free survival is defined as the time between the date of operation and the date of recurrence or metastasis. Overall survival was defined as the time between the date of operation and the date of death or final follow-up.

\section{Statistical Analysis}

Nonparametric data are represented by a median of interquartile range. Chi-square test or Fishers were used to test classification variables and rank-sum test continuous variables accurately and compare baseline and clinical characteristics. Univariate analysis was performed to evaluate that whether changing surgical treatment time (less than or greater than 11 weeks) affects short-term postoperative clinical outcome. The short-term clinical outcome included hospitalization time, anal exsufflation time, anal defecation time, eating liquid diet time, drainage tube extraction time, 30 days readmission, 30 days reoperation, 30 days mortality rate, anastomotic leakage, and other postoperative complications. Logistic analysis was used to analyze the factors affecting pathological complete remission. These factors included CEA level before nCRT, location of tumor before nCRT, $\mathrm{T}$ stage before nCRT, neoadjuvant chemotherapy, positive lymph nodes before nCRT, MRI EMVI $(+)$ and interval time after radiotherapy. The data in the logistic regression analysis were expressed by dominance ratio, 95\% confidence interval, and $P$-value. Kaplan-Meier estimation and Cox proportional regression analysis were used to analyze the three-year disease-free survival (DFS) and overall survival (OS) and draw the survivorship curve and disease-free survival curve. All statistics were completed by SPSS 21.0 software, and $P<0.05$ was statistical significance. 


\section{Results}

\section{Basic Information and Clinical} Characteristics of Patients Before Neoadjuvant Chemoradiotherapy

One hundred and twenty-three patients with locally advanced rectal cancer underwent total mesorectal excision (TME) after neoadjuvant chemoradiotherapy. Among them, there were $81(65.85 \%)$ male patients and $42(34.15 \%)$ female patients. The median age was 56.5 years in SG, and 59 years in LG. There were $13(10.57 \%)$ patients with grade I, $99(80.49 \%)$ patients with grade II, 11 (8.94\%) patients with grade III and no patients with grade IV in the ASA classification of anesthesia. Before nCRT, 57 (46.34\%) patients had normal CEA level and 36 (29.27\%) patients had normal CA19-9 level. The average length of tumors measured by preoperative MRI was $5.19 \mathrm{~cm}$ in $\mathrm{SG}$ and $5.23 \mathrm{~cm}$ in LG, respectively. According to the distance from the anal verge measured by colonoscopy, there were $63(51.22 \%)$ patients with low rectal cancer $(\leq 5 \mathrm{~cm})$ and $60(48.78 \%)$ patients with middle rectal cancer $(6-10 \mathrm{~cm})$. Preoperative colonoscopy showed that 47 (38.21\%) patients had tumors encircling the bowel cavity completely. High-resolution magnetic resonance imaging (MRI) of the pelvis was used in all patients to evaluate the depth of local stage invasion, metastatic lymph nodes, CRM, and EMVI. There were 65 (52.85\%) patients with $\mathrm{T}_{3}$ and 58 (47.15\%) patients with $\mathrm{T}_{4} ; 16(13.01 \%)$ patients with N0, 33 (26.83\%) patients with $\mathrm{N}_{1}, 74(60.16 \%)$ patients with $\mathrm{N}_{2} ; 96(78,05 \%)$ patients with CRM and 77 (62.6\%) patients with EMVI. Preoperative magnetic resonance imaging was performed after nCRT. The degree of tumor regression was assessed according to Mandard mriTRG grading. There was no significant difference in the number of patients with mriTRG $1-4$ between the two groups $(P=0.799)$. The detail is shown in Table 1 . The operation interval is mainly $8-11$ weeks. The specific division is shown in Figure 1.

\section{Preoperative Features and Short-Term Outcome}

No distant metastases occurred in any patients during neoadjuvant chemoradiotherapy. Among them, 78 (63.93\%) patients underwent low anterior resection (LAR), $45(36.07 \%)$ patients underwent abdominoperineal resection (APR) of the rectum, and the sphincter preservation rates of middle and low rectal cancer were $66.7 \%$ and $59.7 \%$, respectively. The prophylactic ileostomy or transverse colostomy
Table I Basic Information and Clinical Characteristics of Patients Before Neoadjuvant Chemoradiotherapy

\begin{tabular}{|c|c|c|c|c|}
\hline & & SG $(n=66)$ & LG $(n=57)$ & $P$-value \\
\hline Sex & $\begin{array}{l}\text { Man } \\
\text { Woman }\end{array}$ & $\begin{array}{l}40(32.52 \%) \\
26(21.14 \%)\end{array}$ & $\begin{array}{l}41(33.33 \%) \\
16(13.01 \%)\end{array}$ & $0.187 c$ \\
\hline Median age & & $\begin{array}{l}56.5 \\
(50-64)\end{array}$ & $\begin{array}{l}59 \\
(50-65.5)\end{array}$ & $0.479 m$ \\
\hline BMI (kg/m2) & & $\begin{array}{l}22.28 \\
(20.37-24.5)\end{array}$ & $\begin{array}{l}22.27 \\
(20.5-24.23)\end{array}$ & $0.817 \mathrm{~m}$ \\
\hline ASA grading & $\begin{array}{l}\text { I } \\
\text { II } \\
\text { III }\end{array}$ & $\begin{array}{l}7(5.69 \%) \\
55(44.72 \%) \\
4(3.25 \%)\end{array}$ & $\begin{array}{l}6(4.88 \%) \\
44(35.77 \%) \\
7(5.69 \%)\end{array}$ & $0.480 c$ \\
\hline CEA & $\geq 5 \mathrm{ng} / \mathrm{L}$ & $31(25.2 \%)$ & $26(21.14 \%)$ & $0.880 c$ \\
\hline CA19-9 & $\geq 27 \mathrm{ng} / \mathrm{L}$ & $27(21.75 \%)$ & $9(7.32 \%)$ & $0.002 c$ \\
\hline $\begin{array}{l}\text { Average length of } \\
\text { tumors }(\mathrm{cm})\end{array}$ & & $5(4-6)$ & $5(4-5.65)$ & $0.984 m$ \\
\hline Tumor location & $\begin{array}{l}\text { Low } \\
\text { Middle }\end{array}$ & $\begin{array}{l}31(25.2 \%) \\
35(28.46 \%)\end{array}$ & $\begin{array}{l}32(26.02 \%) \\
25(20.32 \%)\end{array}$ & $0.3 \mathrm{lc}$ \\
\hline T stage & $\begin{array}{l}\mathrm{T}_{3} \\
\mathrm{~T}_{4}\end{array}$ & $\begin{array}{l}34(27.64 \%) \\
32(26.02 \%)\end{array}$ & $\begin{array}{l}31(25.2 \%) \\
26(21.14 \%)\end{array}$ & $0.75 c$ \\
\hline $\mathrm{N}$ stage & $\begin{array}{l}\mathrm{N}_{0} \\
\mathrm{~N}_{1} \\
\mathrm{~N}_{2}\end{array}$ & $\begin{array}{l}8(6.5 \%) \\
23(18.7 \%) \\
35(28.46 \%)\end{array}$ & $\begin{array}{l}8(6.5 \%) \\
10(8.13 \%) \\
39(31.71 \%)\end{array}$ & $0.095 c$ \\
\hline Clinic stage & $\begin{array}{l}\text { II } \\
\text { III }\end{array}$ & $\begin{array}{l}8(6.5 \%) \\
58(47.15 \%)\end{array}$ & $\begin{array}{l}7(5.69 \%) \\
50(40.65 \%)\end{array}$ & $0.978 c$ \\
\hline CRM & $(+)$ & $55(44.72 \%)$ & $45(36.59 \%)$ & $0.534 c$ \\
\hline EMVI & $(+)$ & $4 \mathrm{I}(33.33 \%)$ & $32(26.02 \%)$ & $0.50 \mathrm{lc}$ \\
\hline nCT & & $58(47.15 \%)$ & $50(40.65 \%)$ & $0.978 c$ \\
\hline MRiTRG & $\begin{array}{l}1 \\
2 \\
3 \\
4\end{array}$ & $\begin{array}{l}4(3.25 \%) \\
27(21.95 \%) \\
30(24.39 \%) \\
5(4.07 \%)\end{array}$ & $\begin{array}{l}4(3.25 \%) \\
20(16.26 \%) \\
26(21.14 \%) \\
7(5.69 \%)\end{array}$ & $0.799 c$ \\
\hline
\end{tabular}

Abbreviations: m, Mann-Whitney; c, chi-square; BMI, body mass index; ASA, American Society of Anesthesiologists; CEA, carcinoembryonic antigen; CAI9-9, carbohydrate antigen 19-9; CRM, circumferential resection margin; EMVI, extramural vascular invasion; $\mathrm{nCT}$, neoadjuvant chemotherapy; MRiTRG, magnetic resonance imaging tumor regression grading; SG, short-interval group; LG, long-interval group; T, tumor; N, nodal.

rate in LAR was $79.5 \%$. Most (113 (91.9\%)) of the operations were laparoscopic assisted, 4 cases were robotic assisted, 5 cases were traditional operation, and 1 case was conversion to open operation due to the difficulty of 


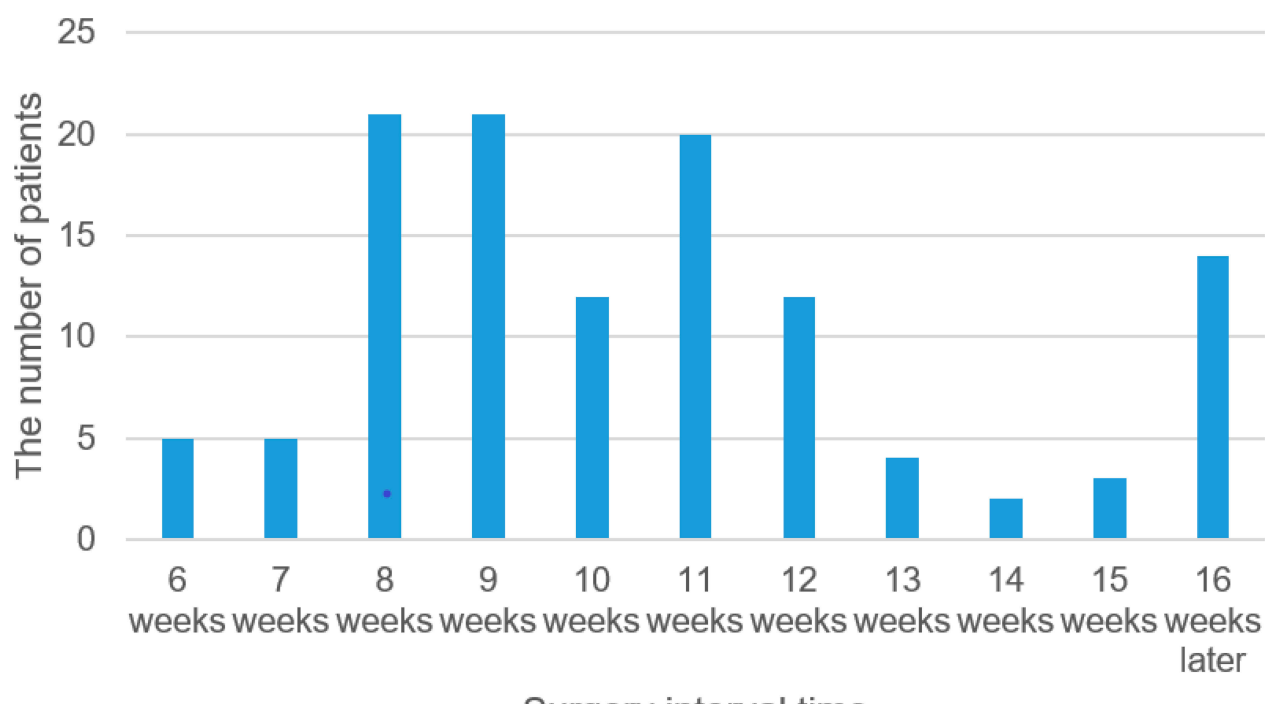

Surgery interval time

Figure I Distribution of patients in different surgery interval times after neoadjuvant chemoradiotherapy.

laparoscopic operation. The median operative time was $240 \mathrm{~min}$ in SG and $285 \mathrm{~min}$ in LG, and the median intraoperative blood loss was $100 \mathrm{~mL}$ in both groups. The median time of anal exsufflation, defecation, and liquid diet was 2 days, 3 days, and 3 days, respectively. The median abdominal drainage volume was $35 \mathrm{~mL}$ in $\mathrm{SG}$ and $30 \mathrm{~mL}$ in $\mathrm{LG}$ on the third day after operation, and the median time of drainage tube extraction was 7 days after operation in both groups. Altogether 33 (26.83\%) patients had complications during hospitalization, including anastomotic leakage (6 cases), abdominal infection (15 cases), postoperative intestinal obstruction ( 8 cases), perineal incision infection (11 cases), rectovaginal leakage (3 cases), urinary retention (5 cases), and urinary tract infection (1 case). Some patients suffered from several complications at the same time, such as anastomotic leakage accompanied by abdominal infection, pulmonary infection, and intestinal obstruction. After discharge, 7 patients suffered from unplanned readmission within 30 days. One patient underwent emergency transverse colostomy because of anastomotic leakage. No patient died within 30 days. Table 2 shows the details.

\section{Pathological Results}

The pathological types of rectal cancer in all patients were adenocarcinoma, including 1 patient with highly differentiated adenocarcinoma, 75 (60.98\%) patients with moderately differentiated adenocarcinoma, $22(17.89 \%)$ patients with poorly differentiated adenocarcinoma and $25(20.33 \%)$ patients with mucinous adenocarcinoma. Postoperative pathological examination showed that there were $22(17.89 \%)$ patients with $\mathrm{T}_{0}$, including 21 patients with negative lymph nodes and 1 patient with positive lymph nodes. Consequently, 21 patients had pathological complete remission, and the pathological complete remission rate was $17.07 \%$. There were 77 patients without lymph node metastasis after nCRT, accounting for $62.6 \%$ in total. The median number of lymph nodes detected was 10 in SG and 8 in LG, separately. The numbers of postoperative pathological stages I, II, and III were 26,32 , and 44, respectively; the downstage in T stage was 71 patients, the downstage in $\mathrm{N}$ stage was 83 patients, and the downstage in TN stage was 73 patients. According to the TRG grading of AJCC, the degree of tumor regression was assessed. There were 21 patients with grade 0,12 patients with grade 1, 43 patients with grade 2,47 patients with grade 3. The details are shown in Table 3. Logistic regression analysis showed that pathological complete remission was not correlated with CEA level before nCRT, location of tumors before nCRT, T stage before nCRT, neoadjuvant chemoradiotherapy, positive lymph nodes before nCRT, MRI EMVI $(+)$ and operation interval after radiotherapy (Table 4).

\section{Oncological Outcome}

Among 123 patients with neoadjuvant chemoradiotherapy, 1 patient in the SG group died within 8 weeks after operation due to other reasons, and 122 patients were included in the tumor outcome analysis at length. There were 94 (77.05\%) patients with adjuvant chemotherapy after the operation, among which there were 50 patients in SG and 
Table 2 Clinical Features of Surgery and Post-Operation Short-Term Outcome

\begin{tabular}{|c|c|c|c|}
\hline & SG $(n=66)$ & LG $(n=57)$ & $P$-value \\
\hline Operation method & & & $0.42 c$ \\
\hline LAR & $44(35.77 \%)$ & $34(27.64 \%)$ & \\
\hline APR & $22(17.89 \%)$ & $23(18.7 \%)$ & \\
\hline Surgery type & & & $0.806 c$ \\
\hline Traditional & $3(2.44 \%)$ & $2(1.63 \%)$ & \\
\hline Laparoscopy & $60(48.78 \%)$ & $53(43.09 \%)$ & \\
\hline Robotic assisted & $2(1.63 \%)$ & $2(1.63 \%)$ & \\
\hline Conversion to traditional & $\mathrm{I}(0,8 \mathrm{I} \%)$ & 0 & \\
\hline LAR preventive stoma & $34(27.64 \%)$ & $28(22.76 \%)$ & $0.582 c$ \\
\hline Median operative time $(\mathrm{mL})$ & $240(209.3-301.5)$ & $285(212.5-355)$ & $0.119 \mathrm{~m}$ \\
\hline Median intraoperative blood loss $(\mathrm{mL})$ & $100(30-122.5)$ & $100(50-200)$ & $0.553 \mathrm{~m}$ \\
\hline Median postoperative exhaust (day) & $2(2-3)$ & $2(2-3)$ & $0.557 \mathrm{~m}$ \\
\hline Median postoperative defecation (day) & $3(2-4)$ & $3(2-3)$ & $0.842 \mathrm{~m}$ \\
\hline Median postoperative liquid diet (day) & $3(2-5)$ & $3(2-4)$ & \\
\hline Median postoperation abdominal drainage volume on the third day after $(\mathrm{mL})$ & $35(10-100)$ & $30(10-100)$ & $0.901 \mathrm{~m}$ \\
\hline The median time of drainage tube extraction & $7(6-9)$ & $7(5-9)$ & $0.424 \mathrm{~m}$ \\
\hline Median time in hospital after operation (day) & $9(7-12.25)$ & $9(7-14)$ & $0.882 \mathrm{~m}$ \\
\hline Postoperation complications & $18(14.63 \%)$ & $15(12.2 \%)$ & $0.905 c$ \\
\hline Anastomotic leakage & $\mathrm{I}(0,8 \mathrm{I} \%)$ & $5(4.07 \%)$ & $0.08 \mathrm{f}$ \\
\hline Abdominal infection & $7(5.69 \%)$ & $8(6.5 \%)$ & $0.562 \mathrm{c}$ \\
\hline Intestinal obstruction & $4(3.25 \%)$ & $4(3.25 \%)$ & $0.83 c$ \\
\hline Urinary retention & $4(3.25 \%)$ & $\mathrm{I}(0,8 \mathrm{I} \%)$ & $0.228 c$ \\
\hline Cardiopulmonary complications & $4(3.25 \%)$ & $\mathrm{I}(0,81 \%)$ & $0.228 c$ \\
\hline Rectovaginal leakage & $\mathrm{I}(0,8 \mathrm{I} \%)$ & $2(1.63 \%)$ & $0.475 c$ \\
\hline Incision infection & $6(4.88 \%)$ & $5(4.07 \%)$ & $0.95 \mathrm{Ic}$ \\
\hline Urinary tract infection & $\mathrm{I}(0,8 \mathrm{I} \%)$ & $\mathrm{I}(0.8 \mathrm{I} \%)$ & $0.917 c$ \\
\hline 30-day readmission & $4(3.25 \%)$ & $3(2.44 \%)$ & $0.849 c$ \\
\hline 30-day mortality & 0 & 0 & \\
\hline 30-day reoperation & $\mathrm{I}(0,8 \mathrm{I} \%)$ & 0 & $0.35 \mathrm{Ic}$ \\
\hline
\end{tabular}

Abbreviations: m, Mann--Whitney; c, chi-square; f, Fisher's exact; LAR, low anterior resection; APR, abdominoperineal resection; SG, short-interval group; LG, longinterval group.

44 patients in $\mathrm{LG}(P=0.972)$. The median follow-up time was 38 months (15-84) in all patients, 39 months (16-84) in the SG group and 38 months $(15-50)$ in LG $(P=0.38)$. Recurrence or metastasis occurred in 36 (29.5\%) patients, 18 patients in SG, and 18 patients in $\mathrm{LG}$, respectively $(\mathrm{P}=0.639)$. The 3 -year disease-free survival was $72.3 \%$ in $\mathrm{SG}$ and $68.4 \%$ in $\mathrm{LG}(P=0.795)$ (Figure $2 \mathrm{~A})$. The 3 -year overall survival of the two groups was very similar, at $83.1 \%$ and $80.7 \%$, respectively $(P=0.824)$, with no statistical significance (Figure 2B). COX analysis showed that recurrence or metastasis after neoadjuvant chemoradiotherapy was correlated with positive lymph nodes $(P<0.05)$, $\mathrm{HR}=4.812$ (95\% CI 2.4-9.648), overall survival time was correlated with positive lymph nodes $(P=0.003), \mathrm{HR}=4.348$ (95\% CI 1.67-11.349) (Table 5).

\section{Discussion}

Currently, the time interval between the last radiotherapy and standard TME operation for rectal cancer after neoadjuvant chemoradiotherapy remains controversial. Most studies believed that the prolongation of operation interval could reduce the local recurrence rate, but precise interval time is still uncertain. ${ }^{8,9,18-21}$ This study prolonged the interval to 11 weeks and analyzed the surgical conditions, postoperative complications, pathological examination results, and oncological outcome.

The Lyon R90-01 randomized-controlled trial divided patients into 2-week and 6-8-week intervals for operation. The results showed that compared with the 2-week group, the 6-8-week group had better tumor regression, downstage, 
Table 3 Post-Operation Pathological Characteristics

\begin{tabular}{|c|c|c|c|}
\hline & SG $(n=66)$ & LG $(n=57)$ & $P$ value \\
\hline $\begin{array}{l}\text { Degree of differentiation } \\
\text { High differentiation } \\
\text { Moderate differentiation } \\
\text { Poor differentiation } \\
\text { Mucinous adenocarcinoma }\end{array}$ & $\begin{array}{l}\mathrm{I}(0,8 \mathrm{I} \%) \\
40(32.52 \%) \\
\mathrm{I}(\mathrm{I} \mid \mathrm{I} .38 \%) \\
\mathrm{II}(8.94 \%)\end{array}$ & $\begin{array}{l}0 \\
35(28.46 \%) \\
8(6.5 \%) \\
14(11.38 \%)\end{array}$ & $0.443 c$ \\
\hline $\begin{array}{c}\text { ypT stage } \\
T_{0} \\
T_{1} \\
T_{2} \\
T_{3} \\
T_{4}\end{array}$ & $\begin{array}{l}12(9.76 \%) \\
2(1.62 \%) \\
20(16.26 \%) \\
17(13.82 \%) \\
15(12.2 \%)\end{array}$ & $\begin{array}{l}10(8.13 \%) \\
0 \\
12(9.76 \%) \\
17(13.82 \%) \\
18(14.63 \%)\end{array}$ & $0.43 \mathrm{lc}$ \\
\hline $\begin{array}{l}\text { ypN stage } \\
\mathrm{N}_{0} \\
\mathrm{~N}_{1} \\
\mathrm{~N}_{2}\end{array}$ & $\begin{array}{l}46(37.4 \%) \\
11(8.94 \%) \\
9(7.32 \%)\end{array}$ & $\begin{array}{l}31(25.2 \%) \\
19(15.45 \%) \\
7(5.69 \%)\end{array}$ & $0.097 \mathrm{c}$ \\
\hline $\begin{array}{l}\text { ypTN tage } \\
0 \\
\text { I } \\
\text { II } \\
\text { III }\end{array}$ & $\begin{array}{l}11(8.94 \%) \\
19(15.45 \%) \\
17(13.82 \%) \\
19(15.45 \%)\end{array}$ & $\begin{array}{l}10(8.13 \%) \\
7(5.69 \%) \\
15(12.2 \%) \\
25(20.33 \%)\end{array}$ & $0.116 c$ \\
\hline $\begin{array}{l}T \text { downstaging }(y \mathrm{p} T<\mathrm{cT}) \\
\mathrm{N} \text { downstaging }(\mathrm{ypN}<\mathrm{cN}) \\
\text { TN downstaging }(\mathrm{yp} T \mathrm{~N}<\mathrm{cTN})\end{array}$ & $\begin{array}{l}4 I(33.33 \%) \\
45(36.59 \%) \\
44(35.77 \%)\end{array}$ & $\begin{array}{l}30(24.39 \%) \\
38(30.89 \%) \\
29(23.58 \%)\end{array}$ & $\begin{array}{l}0.228 c \\
0.858 c \\
0.075 c\end{array}$ \\
\hline $\begin{array}{l}\text { TRG } \\
\text { TRG0 } \\
\text { TRGI } \\
\text { TRG2 } \\
\text { TRG3 }\end{array}$ & $\begin{array}{l}\mathrm{II}(8.94 \%) \\
8(6.5 \%) \\
23(18.7 \%) \\
24(19.51 \%)\end{array}$ & $\begin{array}{l}10(8.13 \%) \\
4(3.25 \%) \\
20(16.26 \%) \\
23(18.7 \%)\end{array}$ & $0.81 \mathrm{lc}$ \\
\hline $\begin{array}{l}\mathrm{PCR}\left(\mathrm{yP} \mathrm{T}_{0} \mathrm{~N}_{0}\right) \\
\text { Number of harvested lymph nodes } \\
\text { Number of positive lymph nodes }\end{array}$ & $\begin{array}{l}11(8.94 \%) \\
10(5-15) \\
0(0-1)\end{array}$ & $\begin{array}{l}10(8.13 \%) \\
8(3-12) \\
0(0-1.5)\end{array}$ & $\begin{array}{l}0.897 \mathrm{c} \\
0.038 \mathrm{~m} \\
0.302 \mathrm{c}\end{array}$ \\
\hline
\end{tabular}

Abbreviations: $m$, Mann--Whitney; c, chi-square; TRG, tumor regression grading; PCR, pathological complete remission; SG, short-interval group; LG, longinterval group; T, tumor; N, nodal; TN, tumor and nodal stage; ypT, pathological tumor stage after neoadjuvant therapy; ypN, pathological nodal stage after neoadjuvant therapy; yPTN, pathological tumor and nodal stage after neoadjuvant therapy; $\mathrm{CT}$, clinic tumor stage; $\mathrm{CN}$, clinic nodal stage; $\mathrm{CTN}$, clinic tumor and nodal stage; $y \mathrm{p} \mathrm{T}_{0} \mathrm{~N}_{0}$, pathological tumor 0 stage and nodal 0 stage after neoadjuvant therapy.

sphincter preservation rate, and pathological complete remission rate. Since then, 6-8 weeks had been considered as the best appropriate time for operation after nCRT. ${ }^{7}$ Prolonging interval time could increase tumor regression, enabling patients to acquire enough neoadjuvant chemotherapy time, which might increase the possibility of pathological complete remission. ${ }^{8,9,18-21}$ Nevertheless, with the prolongation of the interval time, the tissue fibrosis in the radiotherapy area became more serious. Planar fibrosis, severe edema, and local inflammation increased the difficulty of TME operation
Table 4 Influence Factors of Pathological Complete Remission by Multivariate Analysis

\begin{tabular}{|l|l|l|l|l|}
\hline & \multirow{2}{*}{ HR } & \multicolumn{2}{|l|}{$\mathbf{9 5 \%} \mathbf{C l}$} & \multirow{2}{*}{ Pvalue } \\
\cline { 3 - 4 } & & LOW & UP & \\
\hline Operation interval after & 0.984 & 0.375 & 2.585 & 0.975 \\
radiotherapy & & & & \\
CEA level before nCRT & 1.042 & 0.381 & $2.85 \mathrm{I}$ & 0.936 \\
Location of tumor before nCRT & 0.694 & 0.258 & 1.863 & 0.468 \\
T stage before nCRT & 0.882 & 0.334 & 2.33 & 0.801 \\
Positive lymph nodes before & 1.15 & 0.283 & 4.669 & 0.845 \\
nCRT & & & & \\
MRI EMVI (+) before nCRT & 2.343 & 0.828 & 6.628 & 0.109 \\
Neoadjuvant chemotherapy & 1.821 & 0.491 & 6.757 & 0.37 \\
\hline
\end{tabular}

Abbreviations: T, tumor; CEA, carcinoembryonic antigen; EMVI, extramural vascular invasion; nCRT, neoadjuvant chemoradiotherapy; MRI, magnetic resonance imaging; HR, hazard ratio; $\mathrm{Cl}$, confidence interval; LOW, low limitation; UP, up limitation.

and the related risks during operation. ${ }^{17}$ Garcia-Aguilar et al ${ }^{23}$ found that pelvis fibrosis was more severe with an 11-week interval time than with a 6-week interval time after nCRT. The randomized-controlled study of GRECCAR- $6^{13}$ showed that compared with a 7-week interval time, an 11-week interval time after nCRT would increase postoperative complications and the risk of local recurrence, and affect the quality of total mesorectal excision. However, the prolongation of interval time did not mean an increase in surgical difficulties and complications. Our study found that the estimated blood loss during the operation was basically identical when the interval time was prolonged to 11 weeks. Although the pelvis fibrosis gradually increased after 11 weeks, the edema gradually subsided in the radiotherapy area, and the anatomical plane of the surgical area was clear. Therefore, prolonging to 11 weeks did not increase the operation time, intraoperative bleeding, and complications. After nCRT, the rate of sphincter preservation in middle and low rectal cancer would increase. ${ }^{7}$ Huntington et $\mathrm{al}^{24}$ found that the rate of sphincter preservation was not correlated with interval time, but sphincter preservation rate would decrease if the interval time was extended to 60 days. The sphincter preservation rate of middle and low rectal cancer in our study was $66.7 \%$ (SG) and 59.6\% (LG), respectively. It might be inferred that the tumors would not shrink after reaching a certain time point, which is the best time for surgery. Moore et $\mathrm{al}^{10}$ observed that anastomotic leakage and pelvis abscesses happened more frequently when the operation was carried out after an interval time of 44 days. The present study revealed that the incidence of anastomotic leakage in LG was higher than SG. It illustrated that complications after nCRT for rectal cancer had nothing to do with prolongation of interval 

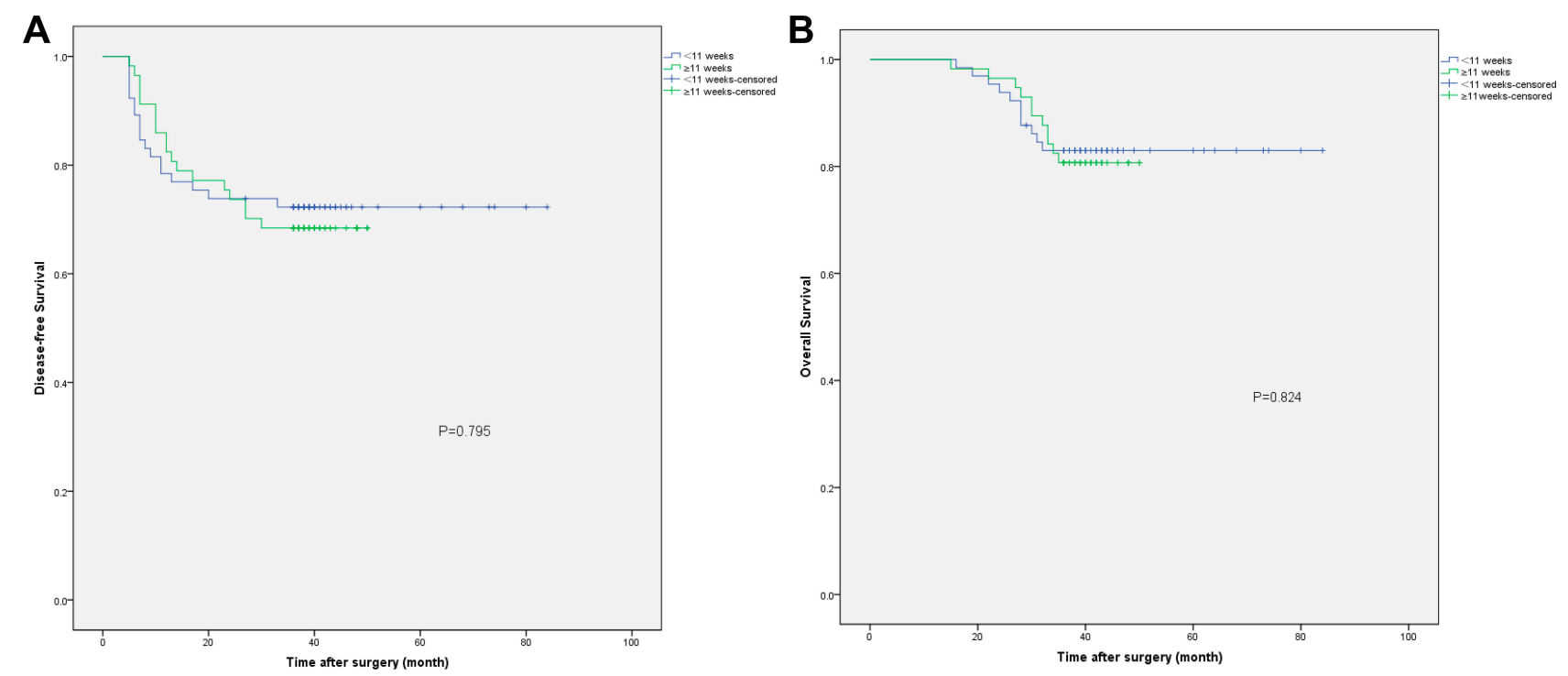

Figure 2 (A) Comparison of disease-free survival between the groups by Kaplan-Meier curves. (B) Comparison of overall survival between the groups by Kaplan-Meier curves.

time $^{25}$. Prolonging interval time could relieve intestinal edema, which was beneficial to getting a clean anatomic planner and better anastomosis. Nevertheless, most patients with sphincter preservation accepted preventive stoma in order to avoid serious postoperative complications, which caused the number of anastomotic leakages observed in the study to be lower than the actual number.

Previous studies have found that DNA destruction of tumor cells mediates apoptosis leading to tumor regression, which is closely related to the interval time. Prolonging the interval time properly could provide better tumor regression and higher pathological complete remission rate. ${ }^{22,28-31}$ It has been reported that about $30 \%$ of rectal tumors did not shrink and $6 \%$ of rectal tumorsgained progression after appropriate nCRT. ${ }^{31}$ The degree of tumor regression is closely correlated with the prognosis of oncology. ${ }^{32,34,35}$ Pathological complete remission indicates a good prognosis. ${ }^{8,9,18-21}$ Sloothaaket et $\mathrm{al}^{33}$ found that the highest PCR rate $(18.0 \%)$ could be obtained by prolonging the operation interval to 10-11 weeks after nCRT. It has been proven that as the interval time increased within 12 weeks, the rate of tumor regression and pCR rate increased gradually, which would decrease over 12 weeks interval time. ${ }^{14}$ De Andrade et $\mathrm{al}^{41}$ performed a study which exhibited that interval time was the sole influencing factor of pathological complete remission,

Table 5 Influence Factors of Overall Survival and Disease-Free Survival After Neoadjuvant Chemoradiotherapy

\begin{tabular}{|c|c|c|c|c|c|c|c|c|}
\hline & \multicolumn{4}{|l|}{ OS } & \multicolumn{4}{|l|}{ DFS } \\
\hline & \multirow[t]{2}{*}{$P$ value } & \multirow[t]{2}{*}{ HR } & \multicolumn{2}{|c|}{$95 \% \mathrm{Cl}$} & \multirow[t]{2}{*}{$P$-value } & \multirow[t]{2}{*}{ HR } & \multicolumn{2}{|c|}{$95 \% \mathrm{Cl}$} \\
\hline & & & LOW & UP & & & LOW & UP \\
\hline Age & 0.296 & 0.977 & 0.936 & 1.02 & 0.2 & 0.979 & 0.948 & 1.011 \\
\hline Gender & 0.743 & 1.179 & 0.44 & 3.159 & 0.467 & 1.319 & 0.626 & 2.78 \\
\hline T stage before nCRT & 0.324 & 0.628 & 0.249 & 1.582 & 0.782 & 0.906 & 0.452 & 1.816 \\
\hline Clinical stage before nCRT & 0.556 & 0.679 & 0.187 & 2.465 & 0.89 & 0.926 & 0.312 & 2.753 \\
\hline Postoperation pathology $\mathrm{T}$ stage & 0.716 & 0.893 & 0.484 & 1.648 & 0.344 & 0.798 & 0.5 & 1.273 \\
\hline Postoperation pathology stage & 0.504 & 0.554 & 0.098 & 3.125 & 0.98 & 1.016 & 0.301 & 3.424 \\
\hline Postoperation lymph nodes $(+)$ & 0.003 & 4.348 & 1.67 & 11.319 & 0 & 4.812 & 2.4 & 9.648 \\
\hline ypTRG & 0.078 & 1.648 & 0.945 & 2.873 & 0.178 & 1.456 & 0.843 & 2.515 \\
\hline Interval time after radiotherapy & 0.651 & 0.809 & 0.322 & 2.029 & 0.592 & 0.824 & 0.406 & 1.671 \\
\hline
\end{tabular}

Abbreviations: OS, overall survival; DFS, disease-free survival; nCRT, neoadjuvant chemoradiotherapy; ypTRG, pathological tumor regression grading after neoadjuvant therapy; T, tumor; HR, hazard ratio; Cl, confidence interval; LOW, low limitation; UP, up limitation. 
and prolonging interval time over 8 weeks would clearly increase the pathological complete remission rate. However, some other studies have shown that prolonging the interval time after nCRT did not increase pCR; instead, this would increase postoperative complications and the occurrence of distant metastasis during the waiting period. ${ }^{13,14}$ Our study showed that the pathological complete remission rate was $16.7 \%(11 / 66)$ in SG and $17.5 \%$ $(10 / 57)$ in LG. Multivariate analysis showed that pCR of tumors had nothing to do with interval time; meanwhile, prolonging interval time did not increase the pCR.

A meta-analysis showed that there was no significant difference in 5-year overall survival and disease-free survival between the group with an interval time within 6-8 weeks and over 6-8 weeks. $^{36}$ Similar conclusions were drawn in our study that 3-year overall survival and diseasefree survival in SG were superior to LG. Appropriate prolongation of interval time could make tumors regress further and provide opportunities for $\mathrm{R}_{0}$ resection, which could reduce local recurrence and distant metastasis as far as possible. However, another study found that the 5-year OS and DFS of patients with interval time over 8-weeks were lower, compared with an interval time of less than 8 weeks. ${ }^{37}$ Prolonging interval time increases pelvis fibrosis, which made it difficult to distinguish the operation plane, increased the difficulty of operation, reduced the quality of TME, decreased chances to complete $\mathrm{R}_{0}$ resection, and increased the risk of local recurrence and distant metastasis; as a result, overall survival and disease-free survival for patients would decline. Erkan et $\mathrm{al}^{38}$ carried out a study which showed that the 5-year overall survival of patients after nCRT with lymph node-negative was better than with lymph node-positive ( 80 vs $86 \%, P=0.014 \mathrm{HR}=1.74,95 \%$ CI 1.33-2.28). It indicated that positive lymph node was a risk factor for oncologic outcome. Studies have reported that lymph node metastasis occurred in $6-17 \%$ of patients with primary tumor complete remission $\left(\mathrm{ypT}_{0}\right)$ after neoadjuvant chemoradiotherapy. ${ }^{39,40}$ Twenty-two patients had complete regression ( $\mathrm{ypT}_{0}$ ) after nCRT in our study, including one patient with positive lymph node in the longinterval group and the rate of positive lymph node was $4.5 \%$. There were two possible reasons for the phenomenon above. In the first place, the radiotherapy dose in the lymphatic drainage area was lower than for the primary tumor. Secondly, the tumor metastasized to lymph nodes because of an interval time that was too long. Survival analysis showed that a positive lymph node was an important influencing factor for 3-year overall survival. The 3-year disease-free survival was also closely correlated with positive lymph nodes. A tumor with positive lymph node was defined as TNM stage III, which had a lower survival rate and higher risks of local recurrence and distant metastasis than tumors with negative lymph node. Therefore, rectal cancer after nCRT with pathological complete regression $\left(\mathrm{ypT}_{0} \mathrm{~N}_{0}\right)$ had a better oncologic outcome.

There are many limitations to this study. Firstly, the study is a single-center retrospective study, which may have selection bias. Although a large amount of basic information is collected, there is information bias still. Secondly, recurrence and metastasis of rectal cancer occurred mostly in the first three years after operation. The follow-up time of most patients was 3 years, which was insufficient to evaluate the prognosis of rectal cancer, including survival rate. Thirdly, postoperative complications were not classified according to ClavienDindo complications, which caused imprecise analysis. Finally, the regimen and times of preoperative and postoperative chemotherapy may affect the pathological results and oncological outcome. We did not compare the regimen and times of chemotherapy between the two groups in the study.

\section{Conclusion}

In conclusion, prolonging the interval time to 11 weeks after neoadjuvant chemoradiotherapy for middle and low rectal cancer does not increase the pathologic complete remission, morbidity, and mortality. There is no significant effect on oncologic outcome after prolonging the interval time to 11 weeks. Therefore, it is safe to prolong interval time to 11 weeks. It is necessary to carry out a larger prospective randomized study so that the precise interval time after nCRT can be confirmed, which will conduct better oncological outcome.

\section{Abbreviations}

BMI, body mass index; CI, confidence interval; DFS, diseasefree survival; TME, total mesorectal excision; nCRT, neoadjuvant chemoradiotherapy; nCT, neoadjuvant chemotherapy; LARC, locally advanced rectal cancer; pCR, pathological complete remission; CRC, colorectal cancer; MRI, magnetic resonance imaging CRM, circumferential resection margin; EMVI, extramural vascular invasion; TRG, tumor regression grading; CEA, carcinoembryonic antigen, CA19-9, carbohydrate antigen 19-9; LAR, low anterior resection; APR, abdominoperineal resection; HR, hazard ratio. 


\section{Data Sharing Statement}

All data generated or analyzed during this study are included in this published article.

\section{Ethics and Consent Statement}

The study was approved by the Institutional Review Board of the First Affiliated Hospital of Chongqing Medical University, and all patients provided their informed consent before starting the treatment. As it was a retrospective clinical study, all the patients were contacted by telephone to obtain verbal informed consent and it was approved by the ethics committee.

\section{Author Contributions}

All authors made substantial contributions to conception and design, acquisition of data, or analysis and interpretation of data; took part in drafting the article or revising it critically for important intellectual content; gave final approval of the version to be published; and agree to be accountable for all aspects of the work.

\section{Disclosure}

The authors report no conflicts of interest in this work.

\section{References}

1. Parkin DM. Global cancer statistics in the year 2000. Lancet Oncol. 2001;2(9):533-543. doi:10.1016/S1470-2045(01)00486-7

2. Enker WE. Total mesorectal excision - the new golden standard of surgery for rectal cancer. Ann Med. 1997;29(2):127-133. doi:10.3109/ 07853899709113698

3. Guillem JG, Chessin DB, Cohen AM, et al. Long-term oncologic outcome following preoperative combined modality therapy and total mesorectal excision of locally advanced rectal cancer. Ann Surg. 2005;241(5):829-838. doi:10.1097/01.sla.0000161980.46459.96

4. Lim CS, Mehigan BJ, Hartley JE, Monson JR. Neoadjuvant therapy in the treatment of high-risk rectal carcinoma. Surg Oncol. 1999;8 (1):1-11. doi:10.1016/S0960-7404(99)00017-1

5. Janjan NA, Khoo VS, Abbruzzese J, et al. Tumor downstaging and sphincter preservation with preoperative chemoradiation in locally advanced rectal cancer: the M. D. Anderson cancer center experience. Int J Radiat Oncol Biol Phys. 1999;44(5):1027-1038.

6. Sauer R, Becker H, Hohenberger W, et al. Preoperative versus postoperative chemoradiotherapy for rectal cancer. $N$ Engl $J$ Med. 2004;351(17):1731-1740. doi:10.1056/NEJMoa040694

7. Francois Y, Nemoz CJ, Baulieux J, et al. Influence of the interval between preoperative radiation therapy and surgery on downstaging and on the rate of sphincter-sparing surgery for rectal cancer: the Lyon R90-01 randomized trial. J Clin Oncol. 1999;17(8):2396. doi:10.1200/ JCO.1999.17.8.2396

8. Maas M, Nelemans PJ, Valentini V, et al. Long-term outcome in patients with a pathological complete response after chemoradiation for rectal cancer: a pooled analysis of individual patient data. Lancet Oncol. 2010;11(9):807-808. doi:10.1016/S1470-2045(10)70172-8

9. Park IJ, You YN, Agarwal A, et al. Neoadjuvant treatment response as an early response indicator for patients with rectal cancer. $J$ Clin Oncol. 2012;30(15):1770-1776. doi:10.1200/JCO.2011.39.7901
10. Moore HG, Gittleman AE, Minsky BD, et al. Rate of pathologic complete response with increased interval between preoperative combined modality therapy and rectal cancer resection. Dis Colon Rectum. 2004;47(3):279-286. doi:10.1007/s10350-003-0062-1

11. Benson AB III, Venook AP, Al-Hawary MM, et al. NCCN clinical practice guidelines in oncology: rectal Cancer. NCCN. 2018;16 (7):874-901.

12. People's Republic of China health and Family Planning Commission, medical and health authority, Chinese Medical Association oncology branch. Chinese colorectal cancer diagnosis and treatment standard (2017 edition). J Chin Surg. 2018;56(4):241.

13. Lefevre JH, Mineur L, Kotti S, et al. Effect of interval (7 or 11 weeks) between neoadjuvant radiochemotherapy and surgery on complete pathologic response in rectal cancer: a multicenter, randomized, controlled trial (GRECCAR-6). J Clin Oncol. 2016;34(31):3773. doi:10.1200/JCO.2016.67.6049

14. Rombouts AJM, Hugen N, Elferink MAG, Nagtegaal ID, de Wilt JH. Treatment interval between neoadjuvant chemoradiotherapy and surgery in rectal cancer patients: a population-based study. Ann Surg Oncol. 2016;23(11):3593-3601. doi:10.1245/s10434-016-5294-0

15. Tulchinsky H, Shmueli E, Figer A, Klausner JM, Rabau M. An interval $>7$ weeks between neoadjuvant therapy and surgery improves pathologic complete response and disease-free survival in patients with locally advanced rectal cancer. Ann Surg Oncol. 2008;15 (10):2661-2667. doi:10.1245/s10434-008-9892-3

16. Erlandsson J, Holm T, Pettersson D, et al. Optimal fractionation of preoperative radiotherapy and timing to surgery for rectal cancer (Stockholm III): a multicentre, randomised, non-blinded, phase 3, non-inferiority trial. Lancet Oncol. 2017;18(3):336-346. doi:10.1016/S1470-2045(17)30086-4

17. Silva GD, Berho M, Wexner S, et al. Histologic analysis of the irradiated anal sphincter. Dis Colon Rectum. 2003;46 (11):1492-1497. doi:10.1007/s10350-004-6800-1

18. Capirci C, Valentini V, Cionini L, et al. Prognostic value of pathologic complete response after neoadjuvant therapy in locally advanced rectal cancer: long-term analysis of 566 ypCR patients. Int J Radiat Oncol Biol Phys. 2008;72(1):99-107. doi:10.1016/j.ijrobp.2007.12.019

19. Valentini V, Stiphout RGPMV, Lammering G, et al. Nomograms for predicting local recurrence, distant metastases, and overall survival for patients with locally advanced rectal cancer on the basis of European randomized clinical trials. J Clin Oncol. 2011;29 (23):3163-3172. doi:10.1200/JCO.2010.33.1595

20. Zorcolo L, Rosman AS, Restivo A, et al. Complete pathologic response after combined modality treatment for rectal cancer and long-term survival: a meta-analysis. Ann Surg Oncol. 2012;19 (9):2822-2832. doi:10.1245/s10434-011-2209-y

21. Martin ST, Heneghan HM, Winter DC. Systematic review and meta-analysis of outcome following pathological complete response to neoadjuvant chemoradiotherapy for rectal cancer. $\mathrm{Br} J$ Surg. 2012;99(7):918-928. doi:10.1002/bjs.8702

22. Wang Y, Cummings B, Catton P, et al. Primary radical external beam radiotherapy of rectal adenocarcinoma: long term outcome of 271 patients. Radiother Oncol. 2005;77(2):126-132. doi:10.1016/j.radonc.2005.09.001

23. Garcia-Aguilar J, Chow OS, Smith DD, et al. Effect of adding mFOLFOX6 after neoadjuvant chemoradiation in locally advanced rectal cancer: a multicentre, Phase 2 trial. Lancet Oncol. 2015;16 (8):957-966. doi:10.1016/S1470-2045(15)00004-2

24. Huntington CR, Boselli D, Symanowski J, Hill JS, Crimaldi A, Salo JC. Optimal timing of surgical resection after radiation in locally advanced rectal adenocarcinoma: an analysis of the national cancer database. Ann Surg Oncol. 2016;23(3):877-887. doi:10.1245/s10434-015-4927-z

25. Campos-Lobato LFD, Geisler DP, Moreira ADL, Stocchi L, Dietz D, Kalady MF. Neoadjuvant therapy for rectal cancer: the impact of longer interval between chemoradiation and surgery. J Gastrointest Surg. 2011;15(3):444-450. doi:10.1007/ s11605-010-1197-8 
26. Wolthuis AM, Penninckx F, Haustermans K, et al. Impact of interval between neoadjuvant chemoradiotherapy and TME for locally advanced rectal cancer on pathologic response and oncologic outcome. Ann Surg Oncol. 2012;19(9):2833-2841. doi:10.1245/ s10434-012-2327-1

27. Tran C-L, Udani S, Holt A, Arnell T, Kumar R, Stamos MJ. Evaluation of safety of increased time interval between chemoradiation and resection for rectal cancer. Am J Surg. 2006;192(6):0-877. doi:10.1016/j.amjsurg.2006.08.061

28. Habr-Gama A, Perez RO, Proscurshim I, et al. Interval between surgery and neoadjuvant chemoradiation therapy for distal rectal cancer: does delayed surgery have an impact on outcome? Int J Radiat Oncol Biol Phys. 2008;71(4):1181-1188. doi:10.1016/j. ijrobp.2007.11.035

29. Berger C, De MA, Garaud P, et al. Preoperative radiotherapy (RT) for rectal cancer: predictive factors of tumor downstaging and residual tumor cell density (RTCD): prognostic implications. Int $J$ Radiat Oncol Biol Phys. 1997;37(3):619-627. doi:10.1016/S0360-3016(96) 00577-9

30. Horn A, Morild I, Dahl O. Tumour shrinkage and down staging after preoperative radiation of rectal adenocarcinomas. Radiother Oncol. 1990;18(1):19-28. doi:10.1016/0167-8140(90)90019-S

31. Zeng WG, Zhou ZX, Liang JW, et al. Impact of interval between neoadjuvant chemoradiotherapy and surgery for rectal cancer on surgical and oncologic outcome. J Surg Oncol. 2014;110 (4):463-467. doi:10.1002/jso.23665

32. Mihmanlı M, Kabul Gürbulak E, Akgün İE, et al. Delaying surgery after neoadjuvant chemoradiotherapy improves prognosis of rectal cancer. World J Gastrointest Oncol. 2016;8(9):695-706. doi:10.4251/wjgo.v8.i9.695

33. Sloothaak DAM, Geijsen DE, Van Leersum NJ, et al. Optimal time interval between neoadjuvant chemoradiotherapy and surgery for rectal cancer. $\mathrm{Br} J$ Surg. 2013;100(7):933-939. doi:10.1002/ bjs. 9112
34. Karagkounis G, Thai L, Mace AG, et al. Prognostic implications of pathological response to neoadjuvant chemoradiation in pathologic stage III rectal cancer. Ann Surg. 2019;269(6):1117-1123. doi:10.1097/SLA.0000000000002719

35. De Andrade VA, Leal RF, Fagundes JJ, Coy CS, Ayrizono MD. Neoadjuvant therapy and surgery in rectal adenocarcinoma: analysis of patients with complete tumor remission? J Coloproctol. 2013;33 (4):222-227. doi:10.1016/j.jcol.2013.08.008

36. Petrelli F, Sgroi G, Sarti E, Barni S. Increasing the interval between neoadjuvant chemoradiotherapy and surgery in rectal cancer: a meta-analysis of published studies. Ann Surg. 2016;263(3):458. doi:10.1097/SLA.0000000000000368

37. Sun Z, Adam MA, Kim J, Shenoi M, Migaly J, Mantyh CR. Optimal timing to surgery after neoadjuvant chemoradiotherapy for locally advanced rectal cancer. J Am Coll Surg. 2016;222(4):367-374. doi:10.1016/j.jamcollsurg.2015.12.017

38. Erkan A, Mendez A, Trepanier M, et al. Impact of residual nodal involvement after complete tumor response in patients undergoing neoadjuvant (chemo)radiotherapy for rectal cancer. Surgery. 2019;166(4):648-654. doi:10.1016/j.surg.2019.03.026

39. Hughes R, Glynne-Jones R, Grainger J, et al. Can pathological complete response in the primary tumour following pre-operative pelvic chemoradiotherapy for T3-T4 rectal cancer predict for sterilisation of pelvic lymph nodes, a low risk of local recurrence and the appropriateness of local excision? Int J Colorectal Dis. 2006;21 (1):11-17. doi:10.1007/s00384-005-0749-y

40. Tulchinsky H, Rabau M, Shacham-Shemueli E, et al. Can rectal cancers with pathologic T0 after neoadjuvant chemoradiation (ypT0) be treated by transanal excision alone? Ann Surg Oncol. 2006;13(3):347-352. doi:10.1245/ASO.2006.03.029

41. De Andrade VA, Rodrigues CCS, Franco LR, et al. Neoadjuvant therapy and surgery for rectal cancer. Comparative study between partial and complete pathological response. Arq Gastroenterol. 2016;53(3):163-168. doi:10.1590/S0004-28032016000300008

\section{Publish your work in this journal}

Cancer Management and Research is an international, peer-reviewed open access journal focusing on cancer research and the optimal use of preventative and integrated treatment interventions to achieve improved outcomes, enhanced survival and quality of life for the cancer patient.
The manuscript management system is completely online and includes a very quick and fair peer-review system, which is all easy to use. Visit http://www.dovepress.com/testimonials.php to read real quotes from published authors. 